\title{
Supporting Immersive Location-Based Games on Resource-Constrained Platforms
}

\author{
Katsiaryna Naliuka \\ Trinity College Dublin \\ katsiaryna.naliuka@ndrc.ie \\ Roisin Cotton \\ Trinity College Dublin \\ cottonr@tcd.ie
}

\author{
Tara Carrigy \\ Trinity College Dublin \\ tara.carrigy@ndrc.ie \\ Søren K. Jensen \\ Trinity College Dublin \\ Soren.Jensen@ndrc.ie
}

\author{
Natasa Paterson \\ Trinity College Dublin \\ natasa.paterson@ndrc.ie \\ Mads Haahr \\ Trinity College Dublin \\ Mads.Haahr@cs.tcd.ie
}

\begin{abstract}
This paper is concerned with challenges and solutions related to the use of smartphones as a location-based mobile gaming (LBMG) platform. We present here a summary of our experiences from building a feature-rich story-driven LBMG for the Android platform. The game uses location, handset orientation and marker-less augmented reality to support its core mechanics, which in turn support a strong story line.
\end{abstract}

\section{Categories and Subject Descriptors}

K.8.0 [Computing Milieux]: Personal Computing_games

\section{INTRODUCTION}

Smartphones are emerging as a personal computational platform of significant popularity. The latest generation of handsets (iPhone 4, Android 2 devices, etc.) are equipped with a sophisticated combination of excellent media capabilities (screen, GPU, audio) and considerable processing power and memory. Features such as GPS, compass and camera make smartphones attractive for location-based mobile games (LBMGs), a genre which in digital form has existed for around a decade, but which has yet to reach widespread adoption. Despite the recent advances, challenges remain in relation to the use of smartphones as game platforms:

Battery power is of course crucial to the operation of any location-based mobile game, but smartphones are designed to be feature-rich, rather than power-efficient [2].

User interfaces constitute scarce resources in several regards. Most notably, screen real estate is extremely valuable on a handheld device and must be used carefully.

Storage capacity in the form of RAM is a crucial resource on the current smartphones in relation to games.

Processing power in the form of CPU cycles is also a crucial resource, even despite the relatively powerful processors found in the current generation of smartphones.

Permission to make digital or hard copies of all or part of this work for personal or classroom use is granted without fee provided that copies are not made or distributed for profit or commercial advantage and that copies bear this notice and the full citation on the first page. To copy otherwise, to republish, to post on servers or to redistribute to lists, requires prior specific permission and/or a fee.

$A C E$ '10, 17-NOV-2010, Taipei, Taiwan

Copyright 2010 ACM 978-1-60558-863-6/10/11 ...\$10.00.
Location-dependent information in the classification by Forman et al. means that access to information changes with the location of the device [2]. A particular challenge is that knowledge about the location can vary significantly.

In the next sections we briefly describe the design principles behind our game (Sec. 2) and the technical solutions that we adopted to deal with the above mentioned challenges (Sec. 3). Concluding remarks (Sec. 4) end the paper.

\section{GAME AND STORY DESIGN}

Viking Ghost Hunt (VGH) is a location-based adventure game. In this game the player assumes the role of a paranormal investigator and moves around the city hunting for ghosts and solving the mysteries of haunted Viking Dublin (800-1169 AD). The game can be considered a hybrid of a mystery game and Gothic ghost story. Importantly, it takes place in locations that are of direct historical significance to the material and which are chosen to help the player achieve a high degree of immersion. The game is story-driven in the sense that, in order to progress the game, the player must unlock a sequence of distributed, locationspecific, story fragments, which together make up a meaningful story arc. Hence, the VGH story, which is mapped onto actions and space, is revealed through the players' activities as they move through the locations and, in order to preserve the aesthetics of the role-play, these activities are presented in the context of paranormal investigation. In this form of storytelling, the player is not an external observer, but becomes an agent, who advances in the game and progresses the story by interacting with narrative elements, such as location, character and plot line. The game progress is enabled by the following core mechanics:

Navigation Mechanics The player must identify and move to a particular haunting as marked on a map.

Search Mechanics Once within the haunted area, the player must use the paranormal radar to find manifestations of ghosts in the form of audio and visuals (see Fig. 1).

Scan/Capture Mechanics Once a ghost has manifested visually, the player must take photos of it as evidence. The audio manifestations must be recorded and decoded. Once the evidence is collected new ghost manifestations appear. In this way the player unlocks new parts of the story. 
Paranormal activity also manifests itself through the background soundscape that creates the atmosphere of a haunted place. Its intensity varies through the mission. For detailed discussion of sound and game design please see $[3,1]$.

\section{TECHNICAL IMPLEMENTATION}

The design features that have the most impact on the implementation can be summarized as follows. The game is location-based and includes elements of augmented reality. It is also media intensive to support audible and visible ghost manifestations and background sounds, which makes resource management a primary concern. The last feature for consideration is the fact that the game is story-driven, and consequently game missions have a relatively complex structure. In the following, we describe how implementation of these features is affected by the previously enumerated challenges of resource-constrained platforms.

Dealing with GPS Inaccuracy. GPS accuracy in urban environments tends to be mediocre at its best. We employed several strategies to deal with this issue. The most obvious was selecting the areas, in which GPS reception generally tended to be better (e.g., selecting open places rather than those shadowed by trees or buildings). However, since sites of varying character are required to create immersion in the game, this solution turned out to be insufficient and rather unreliable. Another idea that we employed was using regions of complex shapes (rather than simple circles) to trigger the game events. Using polygonal regions allows increasing size of the region, which makes it easier for players to progress through the game, yet at the same time it leaves more freedom for positioning regions without overlapping.

Augmented Reality (AR). The main challenge in AR lies in the anchoring of the image on top of the camera stream. This requires tracking the player's view point either by analyzing the video stream from the camera or using the orientation sensors. Because of the battery and processing power limitations the first approach, to our knowledge, has not been fully implemented on mobile devices till date, except with the use of fiduciaries, which were not an option in our urban environment. We relied on the second approach, mitigating the negative impact of sensor inaccuracy by filtering. In addition we created a form of AR audio by using spatialization, reverberation and binaural recording techniques [3] to realistically match the game's sound effects to those naturally occurring in the environment as way of deliberately creating ambiguity between the game and the environment.

Resource Management. There are two issues in Android that complicate resource management. First, even though devices have RAM modules of impressive capacity the limit on memory available for each application is relatively low (16 Mb in our targeted version of Android). Therefore early preloading of the resources into memory is undesirable. At the same time, the processing power of the handsets remains comparatively low. In Android this problem is exacerbated by the fact that applications must be developed in Java, which involves the use of a virtual machine. The implications are that loading resources takes relatively long. The outcome is that loading resources in parallel with any computation-heavy routine can render the device unresponsive. The solution we adopted was to load the necessary multimedia content before any costly activities take place but to keep it in memory for as short a time as

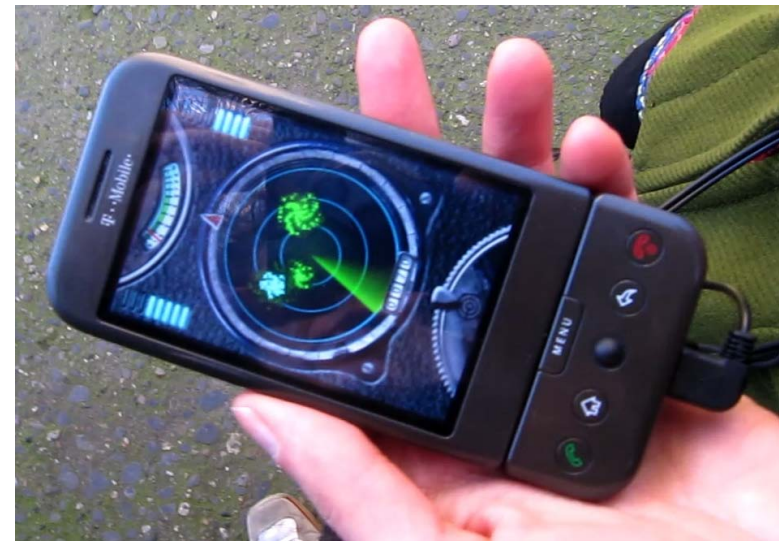

Figure 1: Using paranormal radar to find ghosts

possible. A modular structure for game missions discussed further provided the possibility to implement this approach.

Story Implementation. While location-awareness lends itself well to immersion by placing the player in a physically relevant context, a significant challenge remains with regard to structuring the story content in a fashion that allows it to be presented adaptively in response to changes in user location as well as other modes of interaction. To address this challenge, we have developed a flexible architecture that allows non-linear stories to be expressed and presented as part of a location-based gaming experience. The developed mission infrastructure supports decomposition of the story into atomic location-specific fragments and allows specifying the relations between the individual fragments to ensure that even a complex story with multiple narrative branches is presented to the player in a meaningful way.

\section{CONCLUSION}

This paper has presented our experiences developing an immersive location-based game running purely on a commercially available smartphone platform. We described the challenges of developing such a content-rich and featurerich application in a resource-constraint environment and presented details on the way in which we dealt with these challenges. To us, it is clear that the current generation of smartphones is ready for a new class of games, but that they still constitute a resource-constrained environment and that care must be taken in addressing the associated challenges.

\section{ACKNOWLEDGMENTS}

We are grateful to the National Digital Research Centre (NDRC) for funding the work described in this paper.

\section{REFERENCES}

[1] T. Carrigy, K. Naliuka, N. Paterson, and M. Haahr. Viking Ghost Hunt: Design and evaluation of a location-based mobile game. In Proc. of NordiCHI, 2010.

[2] G. H. Forman and J. Zahorjan. The challenges of mobile computing. IEEE Computer, 27(4):38-47, 1994.

[3] N. Paterson, F. Conway, T. Carrigy, S. K. Jensen, K. Naliuka, and M. Haahr. Implementation and evaluation of audio for a location based augmented reality game. In Proc. of ACM Fun and Games, 2010. 